\title{
The role of the oxide shell in the chemical functionalization of plasmonic Gallium nanoparticles
}

\author{
S. Catalán-Gómez*a , M. Briones ${ }^{\mathrm{b}}$, A. Redondo-Cubero ${ }^{\mathrm{a}}$, F.J. Palomares ${ }^{\mathrm{c}}$, F. Nucciarelli ${ }^{\mathrm{a}}$, E. \\ Lorenzo ${ }^{\mathrm{b}}$ and J.L. Pau ${ }^{\mathrm{a}}$ \\ ${ }^{a}$ Grupo de Electrónica y Semiconductores, Dept. of App. Phys., Autonomous Univ., Cantoblanco, \\ Madrid, Spain E-28049; \\ ${ }^{\mathrm{b}}$ Dept. of Analytical Chem. and Inst. Analysis, Autonomous Univ., Cantoblanco, Madrid, Spain E- \\ 28049; \\ ${ }^{\mathrm{c}}$ Instituto de Ciencia de Materiales de Madrid, CSIC, Cantoblanco, Madrid, Spain E-28049
}

\begin{abstract}
Plasmonic Ga nanoparticles (NPs) were thermally oxidized at low temperature in order to increase the native $\mathrm{Ga}_{2} \mathrm{O}_{3}$ shell thickness and to improve their stability during the chemical functionalization. The optical, structural and chemical properties of the oxidized NPs have been studied by spectroscopic ellipsometry, scanning electron microscopy, grazing incidence X-ray diffraction and X-ray photoelectron spectroscopy. A clear redshift of the peak wavelength is observed with the increasing annealing time due to the $\mathrm{Ga}_{2} \mathrm{O}_{3}$ thickness increase, and barely affecting the intensity of the plasmon resonance. This oxide layer enhances the stability of the NPs upon immersion in ethanol or water. The surface sensitivity properties of the as-grown and oxidized NPs were investigated by linking a thiol group from 6-Mercapto-1-hexanol through immersion. Ellipsometric spectra at the reversal polarization handedness (RPH) condition are in agreement with the Langmuir absorption model, indicating the formation of a thiol monolayer on the NPs.
\end{abstract}

Keywords: Gallium, plasmonics, nanoparticles, ellipsometry, oxidation, functionalization, $\mathrm{MCH}$.

\section{INTRODUCTION}

Biosensing have attracted a great deal of attention in the last decades. The development of fast, sensitive and affordable methods for sensing biomolecules has been the subject of intense research to date. Plasmonics plays an important role for this approach because it provides an optical transduction method from chemical to optical signal in a very quickly and accurately manner ${ }^{1}$. So far, most of the sensors have been developed using different coupling systems such as prisms and gratings. The use of nanostructures can enlarge the sensitivity of these systems, as well as reduce the size significantly ${ }^{2}$. When polarized light at a specific wavelength interact with metallic nanoparticles, collective oscillations of free electrons are created in the vicinity of the surface. This property is well known as localized surface plasmon resonance (LSPR). So far, the plasmonic properties of gold $(\mathrm{Au})$ and silver $(\mathrm{Ag}) \mathrm{NPs}$ have been widely exploited ${ }^{3}$, but they are typically restricted to the infrared (IR) and visible regions ${ }^{4}$. In order to extend this spectral range to the ultraviolet (UV), some alternative materials such as magnesium, aluminum, indium and gallium $(\mathrm{Ga})$ have been studied to have a strong resonance in this region ${ }^{5}$.

Ga NPs are grown by a simple, fast and cost-effective manner using thermal evaporation at low substrate temperatures, which makes them compatible with many substrates such as copper, quartz, glass, silicon ( $\mathrm{Si}$ ), plastics or sapphire, and surfaces modified with 2D materials. Moreover, this bottom-up method is suitable for large area applications. Their most interesting properties are the wide tunability from the IR to the UV varying their size ${ }^{6}$, the spectral splitting of its resonant modes due to the hemispherical geometries and the dependence of the oscillatory modes on the polarization of the incident light ${ }^{7}$.

Optical Sensors 2017, edited by Francesco Baldini, Jiri Homola, Robert A. Lieberman,

Proc. of SPIE Vol. 10231, 102310D · C 2017 SPIE · CCC code: 0277-786X/17/\$18

doi: $10.1117 / 12.2265665$

Proc. of SPIE Vol. 10231 102310D-1 
When NPs are exposed to air, a $0.5-3 \mathrm{~nm}$ thick passivating layer of gallium oxide $\left(\mathrm{Ga}_{2} \mathrm{O}_{3}\right)$ is formed preserving the liquid $\mathrm{Ga}$ from the environment ${ }^{8}$. This shell keeps the liquid $\mathrm{Ga}$ in a supercooled state ${ }^{9}$. The formation of the $\mathrm{Ga}_{2} \mathrm{O}_{3}$ shell is therefore essential for the actual plasmonic response and stability of Ga NPs, but little attention has been paid to it so far. $\mathrm{Ga}_{2} \mathrm{O}_{3}$ is a semiconductor whose wide band gap enables its use as transparent semiconducting oxide because of its low absorbance in a wide spectral range from the middle $\mathrm{UV}$ to the middle $\mathrm{IR}^{10}$. Its most stable crystalline phase is monoclinic $\beta-\mathrm{Ga}_{2} \mathrm{O}_{3}$, which has a low enthalpy of formation compared to other metal oxides $(-1089.1 \mathrm{~kJ} / \mathrm{mol})^{11}$. In the case of liquid Ga NPs, no crystalline phase has been detected for the native oxide layer and the thickness of such layer is assumed to be self-limited by the attenuation of oxygen diffusion ${ }^{11}$.

Despite the unintentional formation of the native oxide, the Ga NPs have exhibited good properties for the enhancement of Raman scattering ${ }^{12}$, for solid-liquid phase change memory elements ${ }^{13}$ and for waveguiding ${ }^{14}$. Regarding biosensing, our group has been widely investigating the detection of different biomolecules such as glutathione ${ }^{15}$, Helicobacter pylori and specific gene mutation related to disease such as cystic fibrosis ${ }^{16}$. These works analyzed the optical properties of the Ga NPs by spectroscopic ellipsometry (SE) before and after the functionalization. For this, the imaginary part of the pseudodielectric function $\left(<\varepsilon_{2}>\right)$ is measured at a specific angle where the p-component of the reflected beam retards with respect to the s-component. This condition is commonly known as reversal of polarization handedness (RPH) and strongly improves the sensitivity of the system for detecting molecules ${ }^{17}$.

In this work, we have performed thermal oxidations onto the Ga NPs in order to increase the $\mathrm{Ga}_{2} \mathrm{O}_{3}$ thickness. The role of this oxide shell in the plasmon resonance has been investigated by spectroscopic ellipsometry. Furthermore, other properties such as stability and chemical functionalization affinity has been studied in order to figure out what would be the effect for future biosensing purposes.

\section{EXPERIMENTAL}

Ga NPs are deposited by Joule-effect thermal evaporation using a vertical Edwards E306 system operating in high vacuum conditions (base pressure of $2 \times 10^{-7} \mathrm{mbar}$ ). Being the size of the NPs limited by the amount of Ga, a fixed mass of $340 \mathrm{mg}$ of Ga (Goodfellow $99.9999 \%$ purity) was chosen in order to attain NPs with a mean radius of about $60 \mathrm{~nm}$. The evaporation process has been carried out under $50 \mathrm{~W}$ power, applied to a tungsten filament (99.90\% purity). The working pressure was $1.5 \times 10^{-5}$ mbar during evaporation. Si (100) substrates were placed $200 \mathrm{~mm}$ away from the metal source. The substrate holder is ice-cooled to avoid surface migration and coalescence of the droplets by unintentional heating.

Thermal treatments of the NPs were performed at atmospheric pressure in a horizontal quartz tube connected to a gas supply line with a QuadraTherm mass flow meter. The temperature inside the furnace is monitored by a proportionalintegral-derivative (PID) controller. For the oxidation treatments, a constant flow of 80 standard cubic centimetres $(\mathrm{sccm})$ of $\mathrm{O}_{2}$ was used. Annealing of each sample was performed under a fixed temperature of $300{ }^{\circ} \mathrm{C}$, but varying time from $15 \mathrm{~min}$ up to $1200 \mathrm{~min}$. After the process, the samples were extracted from the tube, cooling down in ambient conditions. In addition, a reference $\beta-\mathrm{Ga}_{2} \mathrm{O}_{3}$ sample was prepared from $99.999 \%$ powder material and compacted under a compressive load to form a disk with a diameter of $7 \mathrm{~mm}$ and a thickness of $2 \mathrm{~mm}$. It was then annealed in ambient air at $1200{ }^{\circ} \mathrm{C}$ for $3 \mathrm{~h}$.

The optical properties of the samples were analysed by spectroscopic ellipsometry (SE), using a Jobin Yvon UVISEL system equipped with a Xe lamp (1.5-4.5 eV energy range), a $0^{\circ}$ modulator and a $45^{\circ}$ analyser. The measurements are taken in external reflection configuration at $70^{\circ}$ incidence angle referred to the normal of the sample surface. The pseudodielectric constants of the material were obtained from the ellipsometric parameters psi $(\psi)$ and delta $(\Delta)^{18}$.

Scanning electron microscope (SEM) was used to study the morphology of the Ga NPs. The microscope is a FEI XL30SFEG system, operating with $10 \mathrm{keV}$ electron beam with a nominal lateral resolution of $4 \mathrm{~nm}$, being the secondary electrons collected and analysed with an Everhart-Thornley detector.

The crystal structure of the samples has been analysed by grazing incidence X-ray diffraction (GIXRD) using a X'Pert Pro Panalytical system. The incident beam is produced in a $\mathrm{Cu}$ X-ray tube, focused by a Göbel mirror and collimated in a 
Soller slit $(0.04 \mathrm{rad})$. The diffracted beam passes through a $0.18^{\circ}$ parallel plate collimator, a graphite $(002)$ monochromator, and a Soller slit $(0.04 \mathrm{rad})$ before being detected with a Xe gas scintillator. The incident angle was fixed at $0.5^{\circ}$ and the $2 \theta$ angle was varied from $10^{\circ}$ up to $90^{\circ}$, with a step of $0.04^{\circ}$ and an acquisition time of $4 \mathrm{~s}$ per point.

X-ray photoelectron spectroscopic (XPS) has been used to characterize the chemical composition of Ga NPs deposited on Si wafer substrates. XPS spectra were acquired in an UHV chamber with a base pressure of $10^{-10}$ mbar equipped with a hemispherical electron energy analyzer (SPECS Phoibos 150 spectrometer) and a 2-D delay-line detector, using a monochromatic Al- $\mathrm{K}_{\alpha}(1486.74 \mathrm{eV}) \mathrm{X}$-ray source. High resolution spectra were recorded at normal emission using an energy step of $0.025 \mathrm{eV}$ and a pass-energy of $20 \mathrm{eV}$, which provide an overall instrumental peak broadening of $0.45 \mathrm{eV}$. The absolute binding energies of the photoelectron spectra were determined by referencing to the $\mathrm{Si} 2 \mathrm{p}_{3 / 2}$ transition at $99.0 \mathrm{eV}$. In order to compensate for the built up charge on the reference sample of $\mathrm{Ga}_{2} \mathrm{O}_{3}$, a flood gun (FG $15 / 40$, SPECS) has been applied with low energy electrons of $2 \mathrm{eV}$ and $2.12 \mu \mathrm{A}$. The spectra were analyzed with the program CasaXPS ${ }^{19}$ using a Shirley background.

The functionalization of the NPs was carried out with a thiol monolayer for both the as-grown and 400 min oxidized samples. For this purpose, the samples are immersed in a solution of 6-Mercapto-1-hexanol (MCH) in ethanol (EtOH) with concentrations varying from $0.5 \mathrm{mM}$ up to $100 \mathrm{mM}$. One sample for each concentration. MCH was of reagent grade quality obtained from Sigma-Aldrich Co., and used as received without further purification. After 5 hours, the samples are rinsed with EtOH in order to remove non-linked molecules and blown with dry nitrogen. The functionalization was verified by SE at the RPH condition. The maximum slope of the $\left\langle\varepsilon_{2}>\right.$ is extracted from the differentiation with respect to energy and the fit with a Gaussian function. Changes in this maximum value $(\delta \theta)$ with respect to its value before functionalization are used to monitor the concentration sensitivity. The value of the $\delta \theta$ is the average of 6 different points of each sample being the error bars equal to the standard deviation.

\section{RESULTS}

Ga NPs merge during the growth due to coalescence or coarsening processes, sometimes ascribed to the Ostwald ripening mechanism. As a result, for long times, a bimodal size distribution is obtained, with large NPs surrounded by smaller ones. Figure 1(a) shows the result of the evaporation under our experimental conditions, resulting in hemispherical NPs with a mean radius of about $60 \mathrm{~nm}$. After the thermal treatments described previously, the SEM characterization does not show significant changes in size. At $300^{\circ} \mathrm{C}$, the SEM image (Figure 1(b)) shows a kind of a local deformation on top of the NPs. Although, it is not the aim of this work, we have performed an investigation of the origin of these structures varying temperature, time and cooling rate. The results (not shown) suggest that this local deformation is a consequence of the abrupt cooling temperature change when samples are extracted from the furnace. The main point is the space that the liquid Ga leaves within the shell. At quick cooling rates, the shell does not have time to recover elastically and fill the space giving the structures shown in Figure 1(b).

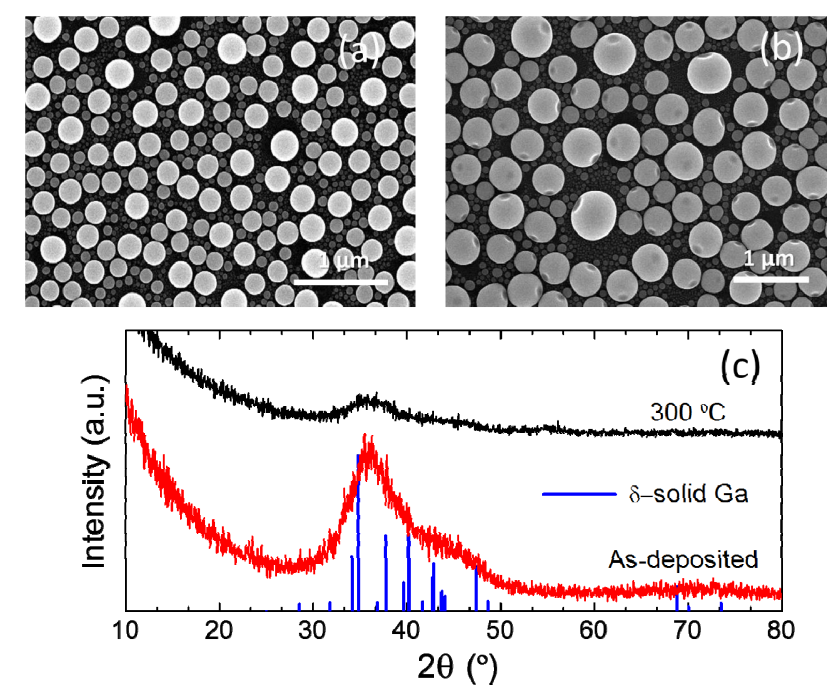

Figure 1. SEM images of (a) as-grown sample (b) $300{ }^{\circ} \mathrm{C}$ oxidized sample for 15 min. (c) GIXRD spectra of both samples with diffraction lines for $\delta$-solid Ga. 
The GIXRD analysis of the NPs is shown Figure 1(c). Firstly, the as-grown sample does not show any narrow peaks regarding crystalline phases. This demonstrates the amorphous character of the $\mathrm{Ga}_{2} \mathrm{O}_{3}$ native shell. The double band presented around 35 and $45^{\circ}$ is due to the presence of a short order range of Ga within the NPs ${ }^{9}$. This double band matches the $\delta$ - phase lines of solid Ga. Secondly, the diffractrogram of the sample intentionally oxidized at $300{ }^{\circ} \mathrm{C}$ shows not only absence of narrow peaks but also lower intensity of the double band, revealing a decrease in the Ga concentration within the NPs. This two results corroborate the increase of the amorphous $\mathrm{Ga}_{2} \mathrm{O}_{3}$ shell thickness.

The plasmonic properties of the Ga NPs has been studied by SE. The $\left\langle\varepsilon_{2}\right\rangle$ is plotted as a function of wavelength in Figure 2. For the as-grown sample, the maximum of the out-of-plane of the LSPR is located around $290 \mathrm{~nm}$ while the inplane resonance is in the middle IR, out of the range of our experimental setup. When thermal treatments are performed at different times, the peak wavelength shifts to longer wavelengths (lower energies). In the literature, a redshift is commonly ascribed to several reasons such as an increase of the NP size ${ }^{20}$, coupling of the resonance with other materials $^{21}$, change of chemical properties of the dissolution where the NPs are ${ }^{5}$, a functionalization with biomolecules ${ }^{1}$, etc. In our case, the redshift is caused by an increase of the refractive index around the metal surface due to the $\mathrm{Ga}_{2} \mathrm{O}_{3}$ thickness increase. It is important to notice that the redshift varies in a non-linear way with time. In addition, the intensity of the plasmon resonance is also affected by the oxide producing the attenuation of the signal due to the dielectric character of the oxide. However, this attenuation is not as intense as for $\mathrm{Ag}^{22}$ and $\mathrm{Al}^{23}$. This is due to the lower extinction coefficient of $\mathrm{Ga}_{2} \mathrm{O}_{3}$ compared to the other oxides ${ }^{24}$. Given these advantages, oxidation processes could offer a good strategy for tuning the plasmon resonance without affecting the plasmonic response significantly.

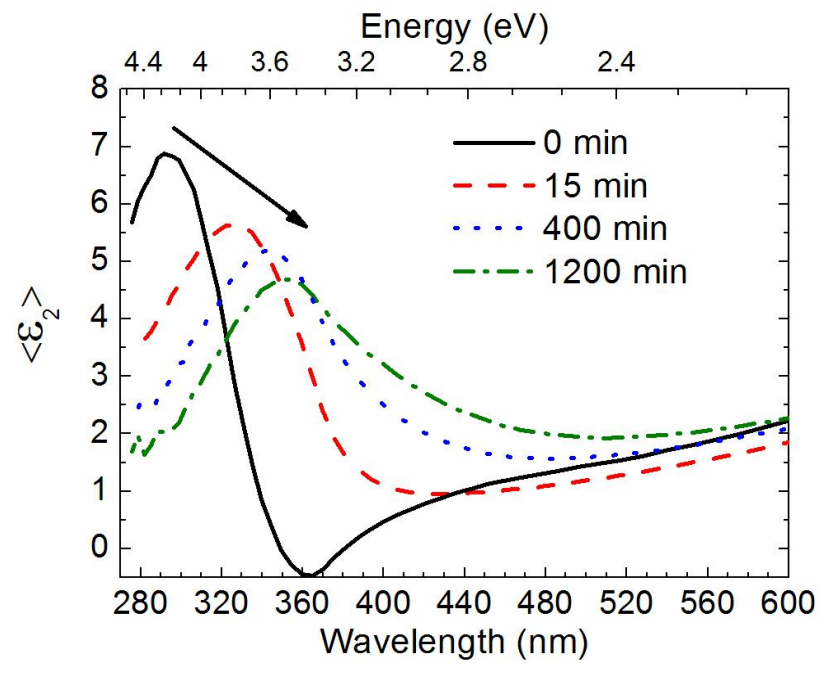

Figure 2. $\left\langle\varepsilon_{2}>\right.$ obtained by SE measurements of samples at $300{ }^{\circ} \mathrm{C}$ for different times.

In order to chemically verify the increment of the oxide and estimate the $\mathrm{Ga}_{2} \mathrm{O}_{3}$ thickness, ex-situ XPS measurements were carried out after the thermal processes. In the survey scan (not shown) Ga 2p, 3s, 3p, and $3 \mathrm{~d}$ electronic levels can be observed. For a detailed chemical analysis, the $3 \mathrm{~d}$ level was selected because of its low binding energy value that provides a higher sampling depth.

The spectra from the oxidized samples together with the as-grown sample is presented in Figure 3. Also, a reference sample of $\mathrm{Ga}_{2} \mathrm{O}_{3}$ has been analysed. Two main peaks can be differentiated for the as-grown sample. The lower binding energy peak is centered at $18.7 \mathrm{eV}$ and it is due to the emission of electrons from the $\mathrm{Ga}$ atoms in a metallic environment ${ }^{25}$. This $3 \mathrm{~d}$ peak can be resolved in a doublet thanks to the fine energy step used $(0.025 \mathrm{eV})$. On the other hand, the upper binding energy peak centered at $21 \mathrm{eV}$ is ascribed to an oxide environment ${ }^{26}$.

As time increases, the signal from the metallic Ga decreases, vanishing at $400 \mathrm{~min}$. These results clearly evidence the oxidation of the NPs. The absence of signal for oxidation times higher than $400 \mathrm{~min}$ does not mean that there is no liquid $\mathrm{Ga}$, but the sampling depth is lower than the oxide shell. Thus, the $\mathrm{Ga}_{2} \mathrm{O}_{3}$ thickness is at least equal to the scape depth of the electrons at this binding energy. In the literature, the scape depth is assumed to be three times the distance of the 
inelastic mean free path. Consequently, for oxidation times higher than $400 \mathrm{~min}$, the oxide has become at least $9 \mathrm{~nm}$ thick.

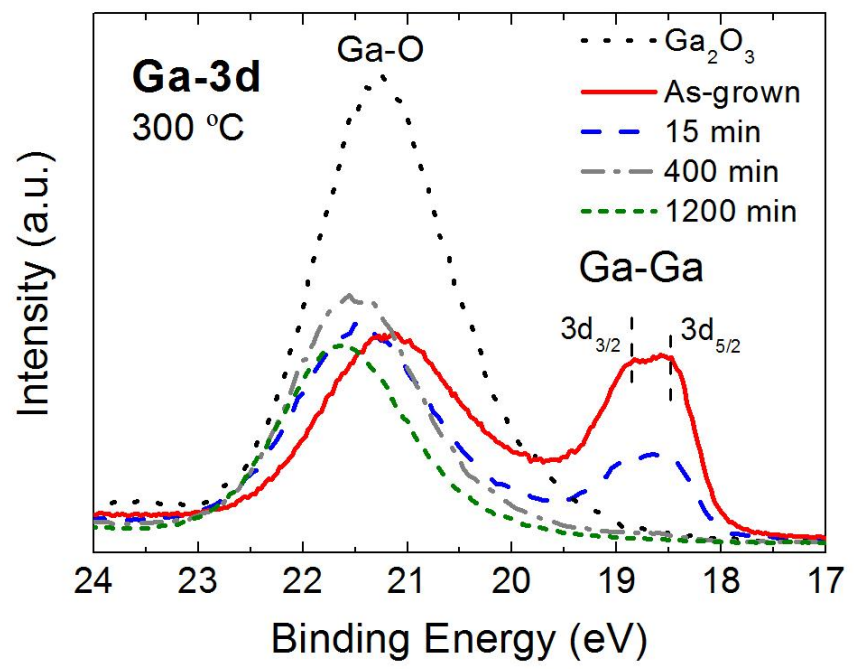

Figure 3. XPS spectra of the $3 \mathrm{~d}$ level of $\mathrm{Ga}$ for samples oxidized at $300{ }^{\circ} \mathrm{C}$ for different times. The data for the as-grown sample and the bulk $\mathrm{Ga}_{2} \mathrm{O}_{3}$ reference sample are also included.

So far we have clearly established that the LSPR of Ga NPs can be tuned dozens of nanometers by increasing the native oxide thickness a few nanometers through thermal oxidations at low temperatures, which does not affect significantly the intensity of the plasmon resonance. Now, we can focus on the effect of this thicker $\mathrm{Ga}_{2} \mathrm{O}_{3}$ on the stability and surface sensitivity to functionalization of the Ga NPs.

The native oxide formed protects the liquid Ga from the air and avoid reactions with the environment. Presumably, an increase of the shell would improve the stability of the NPs. For testing it, we have immersed the as-grown and $400 \mathrm{~min}$ oxidized samples in ethanol (EtOH) for 5 hours in ambient conditions. EtOH has been chosen for being the solvent for the functionalization in the next section. We have measured the $\left\langle\varepsilon_{2}>\right.$ before and after the immersion. Figure 4 shows the $\mathrm{SE}$ spectra of the as-grown and the intentionally oxidized sample before and after the immersion. A shift of $\sim 50 \mathrm{meV}$ is visible for the as-grown sample when immersed, while oxidized NPs show no shift. The origin of the shift is likely due to a wet oxidation of the NPs. Indeed, we have found a higher shift $(130 \mathrm{meV})$ in $\mathrm{H}_{2} \mathrm{O}$ immersion (not shown) probably because of its more oxidizing character than EtOH.

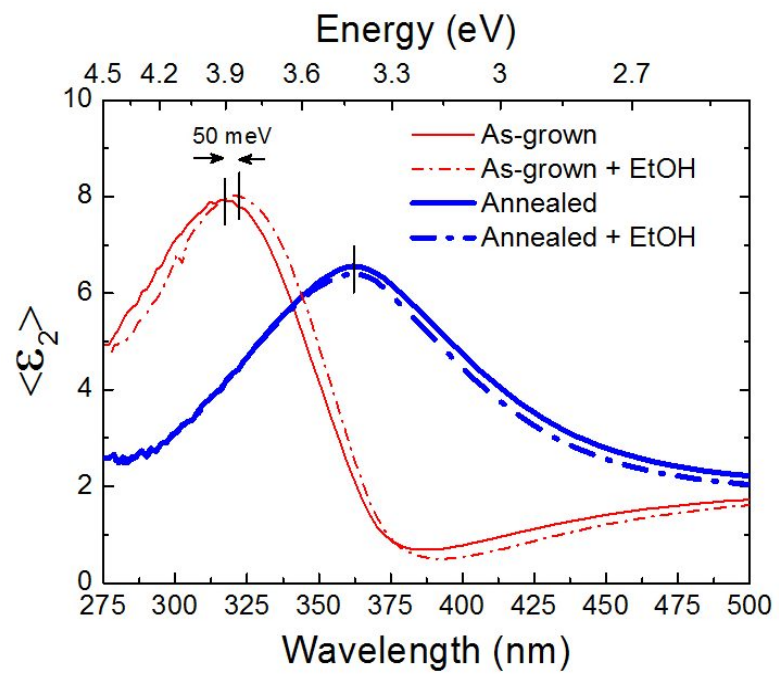

Figure 4. $\left\langle\varepsilon_{2}>\right.$ obtained by SE measurements of samples as-grown and 400 min oxidized before and after immersion in EtOH for $5 \mathrm{~h}$. 
Once the stability has been improved through thermal oxidation, the next characteristic parameter relevant for the biofunctionalization is the surface sensitivity. To analyze this, the samples were functionalized with a 6-Mercapto-1hexanol $(\mathrm{MCH})$ solution in EtOH at different concentrations and left for 5 hours. This gives rise to the chemical modification of the samples with an alcohol termination.

For testing the functionalization, we have measured spectral changes observed in the $\left\langle\varepsilon_{2}>\right.$ under RPH condition. Under this condition, the pseudodielectric function is extremely sensitive to changes in the surrounding medium. This occurs when, at a specific incidence angle, the phase shift difference $(\Delta)$ is $180^{\circ}$ due to the retardation of the s-component with respect to the p-component of the reflected beam ${ }^{17}$. We have optimized the RPH angle for the as-grown and oxidized samples before and after functionalization being $45^{\circ}$ and $52.5^{\circ}$ respectively. This condition gives a characteristic lineshape in the $<\varepsilon_{2}>$ with a maximum peak-to-peak amplitude ${ }^{16}$.

Figure 5(a) shows the SE measurements of a 400 min oxidized sample before and after functionalization with $100 \mathrm{mM}$ of $\mathrm{MCH}$. After the modification with the thiol group, there is a reduction of the peak-to-peak amplitude and a change of the maximum slope. The change of this maximum slope $(\delta \theta)$ has been use as the parameter for tracking the $\mathrm{MCH}$ functionalization as a function of the concentration.

Different concentrations up to $100 \mathrm{mM}$ of $\mathrm{MCH}$ in $\mathrm{EtOH}$ were exposed to the as-grown and 400 min annealed samples. For the as-grown samples, the $\delta \theta$ parameter is not sensitive to the $\mathrm{MCH}$ concentration, and the results evidence a noise pattern (figure not shown). In fact, it is important to note that the $\delta \theta$ due to the immersion in simple EtOH is much higher than the actual change produced by the $\mathrm{MCH}$. Regarding the annealed samples, Figure 5(b) shows a clear tendency of the $\delta \theta$ with the $\mathrm{MCH}$ concentration. As the $\mathrm{MCH}$ increases, a larger $\delta \theta$ is obtained until it reaches a plateau, when the $\mathrm{MCH}$ binding sites on the Ga NPs are saturated. This behavior can be well-described with a Langmuir isotherm function ${ }^{27}$ :

$$
\delta \theta=\frac{\delta \theta_{\max } \cdot K_{L} \cdot c}{1+K_{L} \cdot c}
$$

Where $\delta \theta_{\max }, \mathrm{K}_{\mathrm{L}}$ and $\mathrm{c}$ are the maximum derivative shift, Langmuir constant and MCH concentration, respectively. The fitting, clearly, indicate that the formation of a monolayer is arising as it is explained in the Langmuir absorption $\operatorname{model}^{27}$.
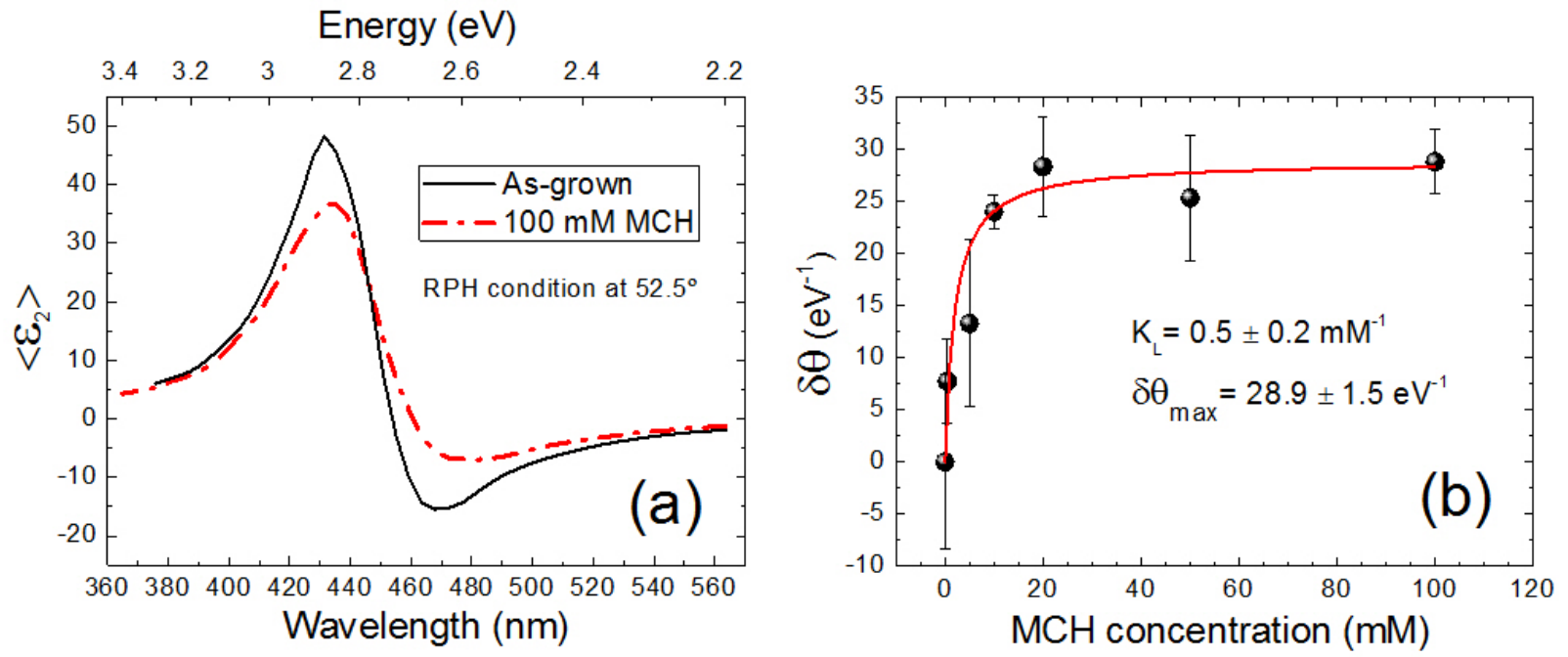

Figure 5. a) $<\varepsilon_{2}>$ at the RPH condition for a 400 min oxidized sample before and after the functionalization of $100 \mathrm{mM}$ of $\mathrm{MCH}$ in EtOH b) change of the maximum slope of the $\left\langle\varepsilon_{2}>\right.$ at the RPH condition after MCH functionalization as a function of the MCH concentration. The red curve corresponds to a Langmuir isotherm fitting whose constant are $\mathrm{K}_{\mathrm{L}}$ and $\delta \theta_{\max }$.

For the sake of comparison, the annealed samples not only have more stability but also they reveal surface sensitivity to a small molecule such as MCH contrary to that missing effect in the as grown samples. Since, at the same time that oxidation treatments could produce a tunable redshift of the peak wavelength, it also improves the surface properties of the Ga NPs. 


\section{CONCLUSIONS}

We have studied the effect of oxidation on the plasmonic properties of the Ga NPs via thermal treatments at low temperature for different times. SE has been used to determine the wavelength of the LSPR of the out-of-plane mode, demonstrating a red shift with the annealing time. XRD measurements show a reduction of the liquid Ga within the NPs, while the increase of the $\mathrm{Ga}_{2} \mathrm{O}_{3}$ thickness has been directly confirmed by XPS measurements. This study opens the possibility of tuning the LSPR of Ga NPs in the UV range by oxidation processes at low temperature without affecting the far-field plasmonic response significantly. Furthermore, the stability of the NPs before and after the oxidation has been studied by immersion in EtOH. SE shows that NPs treated thermally do not experience a shift of the plasmon resonance in the presence of $\mathrm{EtOH}$. Finally, the surface sensitivity has been investigated by functionalization with $\mathrm{MCH}$ through the thiol group. A Langmuir isotherm function relationship is found between the maximum slope of the $<\varepsilon_{2}>$ at the RPH condition and the MCH concentration for the oxidized samples. This proves that the surface of the NPs saturates for high concentrations ( $>20 \mathrm{mM}$ ), explained by the formation of a thiol monolayer. All these results support the use of thermal treatments, not only for tuning the plasmon resonance, but also for improving stability and surface sensitivity of the Ga NPs.

\section{REFERENCES}

[1] Anker, J. N.; Hall, W. P.; Lyandres, O.; Shah, N. C.; Zhao, J.; Van Duyne, R. P., "Biosensing with plasmonic nanosensors" Nat. Mater. 7 (6), $442-453$ (2008).

[2] Aćimović, S. S.; Ortega, M. A.; Sanz, V.; Berthelot, J.; Garcia-Cordero, J. L.; Renger, J.; Maerkl, S. J.; Kreuzer, M. P.; Quidant, R., "LSPR chip for parallel, rapid, and sensitive detection of cancer markers in serum" Nano Lett. 14 (5), 2636-2641 (2014).

[3] Willets, K. A.; Duyne, R. P. V., "Localized surface plasmon resonance spectroscopy and sensing" Annu. Rev. Phys. Chem. 58 (1), 267-297 (2007).

[4] Hubenthal, F.; Ziegler, T.; Hendrich, C.; Alschinger, M.; Träger, F., "Tuning the surface plasmon resonance by preparation of gold-core/silver-shell and alloy nanoparticles" EPJ D 34 (1), 165-168 (2005).

[5] Naik, G. V.; Shalaev, V. M.; Boltasseva, A., "Alternative plasmonic materials: beyond gold and silver" Adv. Mater. 25 (24), 3264-3294 (2013).

[6] Wu, P. C.; Kim, T.-H.; Brown, A. S.; Losurdo, M.; Bruno, G.; Everitt, H. O., "Real-time plasmon resonance tuning of liquid Ga nanoparticles by in situ spectroscopic ellipsometry" Appl. Phys. Lett. 90 (10), 103119 (2007).

[7] Albella, P.; Garcia-Cueto, B.; González, F.; Moreno, F.; Wu, P. C.; Kim, T.-H.; Brown, A.; Yang, Y.; Everitt, H. O.; Videen, G., "Shape Matters: Plasmonic nanoparticle shape enhances interaction with dielectric substrate" Nano Lett. 11 (9), 3531-353 (2011).

[8] Losurdo, M.; Suvorova, A.; Rubanov, S.; Hingerl, K.; Brown, A. S., "Thermally stable coexistence of liquid and solid phases in gallium nanoparticles" Nat. Mater 15 (9), 995-1002 (2016).

[9] Yarema, M.; Wörle, M.; Rossell, M. D.; Erni, R.; Caputo, R.; Protesescu, L.; Kravchyk, K. V.; Dirin, D. N.; Lienau, K.; von Rohr, F.; Schilling, A.; Nachtegaal, M.; Kovalenko, M. V., "Monodisperse colloidal gallium nanoparticles: synthesis, low temperature crystallization, surface plasmon resonance and li-ion storage" J. Am. Chem. Soc. 136 (35), 12422-12430 (2014).

[10] Ortiz, A.; Alonso, J. C.; Andrade, E.; Urbiola, C., "Structural and optical characteristics of gallium oxide thin films deposited by ultrasonic spray pyrolysis" J. Electrochem. Soc. 148 (2), F26-F29 (2001).

[11] Sanz, J. M.; Ortiz, D.; Alcaraz de la Osa, R.; Saiz, J. M.; González, F.; Brown, A. S.; Losurdo, M.; Everitt, H. O.; Moreno, F., "UV plasmonic behavior of various metal nanoparticles in the near- and far-field regimes: geometry and substrate effects" J. Phys. Chem. C 117 (38), 19606-19615 (2013).

[12] Wu, P. C.; Khoury, C. G.; Kim, T.-H.; Yang, Y.; Losurdo, M.; Bianco, G. V.; Vo-Dinh, T.; Brown, A. S.; Everitt, H. O., "Demonstration of surface-enhanced Raman scattering by tunable, plasmonic gallium nanoparticles" J. Am. Chem. Soc. 131 (34), 12032-12033 (2009).

[13] Soares, B. F.; Jonsson, F.; Zheludev, N. I., "All-optical phase-change memory in a single gallium nanoparticle" Phys. Rev. Lett. 98 (15), 153905 (2007).

Proc. of SPIE Vol. 10231 102310D-7 
[14] Krasavin, A. V.; Zheludev, N. I., "Active plasmonics: Controlling signals in Au/Ga waveguide using nanoscale structural transformations" Appl. Phys. Lett. 84 (8), 1416-1418 (2004).

[15] García Marín, A.; Hernández, M. J.; Ruiz, E.; Abad, J. M.; Lorenzo, E.; Piqueras, J.; Pau, J. L., "Immunosensing platform based on gallium nanoparticle arrays on silicon substrates" Biosens. Bioelectron. 74, 1069-1075 (2015).

[16] Marin, A. G.; Garcia-Mendiola, T.; Bernabeu, C. N.; Hernandez, M. J.; Piqueras, J.; Pau, J. L.; Pariente, F.; Lorenzo, E., "Gallium plasmonic nanoparticles for label-free DNA and single nucleotide polymorphism sensing" Nanoscale 8 (18), 9842-9851 (2016).

[17] Verre, R.; Modreanu, M.; Ualibek, O.; Fox, D.; Fleischer, K.; Smith, C.; Zhang, H.; Pemble, M.; McGilp, J. F.; Shvets, I. V., "General approach to the analysis of plasmonic structures using spectroscopic ellipsometry" Phys. Rev. B 87 (23), 235428 (2013).

[18] Tompkins, H. G., [Chapter 1 - Theoretical aspects. In a user's guide to ellipsometry], Academic Press, San Diego, 118 (1993).

[19] Fairley, N., "CASAXPS: Spectrum processing software for XPS, AES and SIMS (Version 2. 3. 14)" CASA Software Ltd. (2006).

[20] Garcia, M. A., "Surface plasmons in metallic nanoparticles: fundamentals and applications" J. Phys. D: Appl. Phys. 44 (28), 283001 (2011).

[21] Niu, J.; Shin, Y. J.; Son, J.; Lee, Y.; Ahn, J.-H.; Yang, H., "Shifting of surface plasmon resonance due to electromagnetic coupling between graphene and Au nanoparticles" Opt. Express 20 (18), 19690-19696 (2012).

[22] Kuzma, A.; Weis, M.; Flickyngerova, S.; Jakabovic, J.; Satka, A.; Dobrocka, E.; Chlpik, J.; Cirak, J.; Donoval, M.; Telek, P.; Uherek, F.; Donoval, D., "Influence of surface oxidation on plasmon resonance in monolayer of gold and silver nanoparticles" J. Appl. Phys. 112 (10), 103531 (2012).

[23] Ziashahabi, A.; Poursalehi, R., "The effects of surface oxidation and interparticle coupling on surface plasmon resonance properties of aluminum nanoparticles as a UV plasmonic material" Procedia Materials Science 11, 434-437 (2015).

[24] Moreno, F.; Albella, P.; Nieto-Vesperinas, M., "Analysis of the spectral behavior of localized plasmon resonances in the near- and far-field regimes" Langmuir 29 (22), 6715-6721 (2013).

[25] Carli, R.; Bianchi, C. L., "XPS analysis of gallium oxides" Appl. Surf. Sci. 74 (1), 99-102 (1994).

[26] Palomares, F. J.; Alonso, M.; Jiménez, I.; Avila, J.; Sacedón, J. L.; Soria, F., "Electron-beam-induced reactions at O2/GaAs(1 0 0) interfaces" Surf. Sci. 1, 482-485, 121-127 (2001).

[27] Langmuir, I., "The adsorption of gases on plane surfaces of glass, mica and platinum" J. Am. Chem. Soc. 40 (9), 1361-1403 (1918). 